\title{
Comparison of Multiple Sets and Single Set of Resistance Training on Muscle Strength and Power
}

Hong $\mathrm{J}^{1}$, Smith $\mathrm{JD}^{2}$, Ross $\mathrm{CN}^{3}$, and Lee $\mathrm{S}^{2}$

${ }^{1}$ The Laboratory of Integrated Physiology; Department of Health \& Human Performance; University of Houston; Houston, TX

${ }^{2}$ Laboratory of Human Performance; Department of Curriculum \& Kinesiology; Texas A\&M University-San Antonio; San Antonio, TX ${ }^{3}$ Department of Art \& Science; Texas A\&M University-San Antonio; San Antonio, TX.

Category: Doctoral

Advisor / Mentor: Lee, Sukho (slee@tamusa.tamus.edu)

ABSTRACT

Studies have been conducted to investigate the effects of different resistance training modalities on muscle strength and power in order to improve physical performance, yet to date findings have been inconclusive. Optimal resistance programs for muscular responses to high and low volume resistance training still need to be explored. The purpose of this study was to compare the effects of multiple sets ( $70 \%$ of 1 RM, 3 sets $/ 10$ reps) and a single set (50-100\% of 1 RM, 1 set/up to 8 reps) of resistance training on muscular strength and power in healthy male college students. A total of 19 students were randomly assigned to either a single set (ST, $n=6)$, multiple sets $(\mathrm{MT}, \mathrm{n}=7)$, or control group $(\mathrm{CON}, \mathrm{n}=6)$. The ST (every 3rd day) and MT (3 times/week) trained for 8 weeks using an inclined leg press. The ST training protocol consists of one set of eight maximum number of repetitions using $50 \%, 75 \%, 90 \%$, and $100 \%$ of $1 \mathrm{RM}$ for the first four repetitions, then needed $10 \mathrm{lbs}$ were added to each subsequent repetition until they failed to lift the weight. Total volume of ST was 147 reps (average 7 reps x 21 days), and MT was 720 reps (30 reps $x 24$ days). One-repetition maximum (1RM), muscle maximal voluntary contraction, peak power, and electromyography were measured at baseline and after 8 weeks of training. Repeated-measured ANOVAs were used to find interaction effect between trial and treatment groups factors. 1RM in all training groups was significantly increased compared to their baseline values $(\mathrm{p}<.001)$. There was no significant difference in $1 \mathrm{RM}$ between the two training groups after 8 weeks of training. Muscle maximal voluntary contraction $(\mathrm{p}<.001)$ and electromyography activity $(\mathrm{p}=.043)$ were significantly increased in MT after 8 weeks of training compared to baseline, but not in ST ( $p=.089$ and $p=.206$, respectively). There was no significant increase on peak power after 8 weeks of resistance training both in MT $(\mathrm{p}=.286)$ and ST $(\mathrm{p}=.372)$. ST, a low volume progressive single set resistance training protocol, is as effective as traditional high volume multi sets training protocol (MT) for increasing muscle strength.

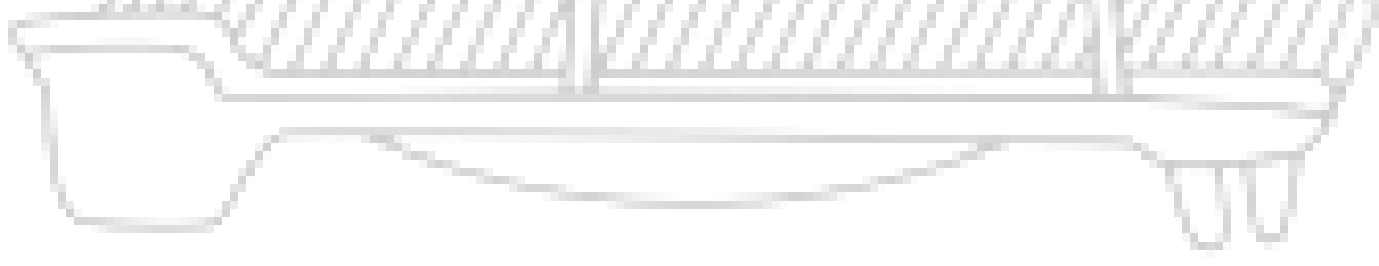

